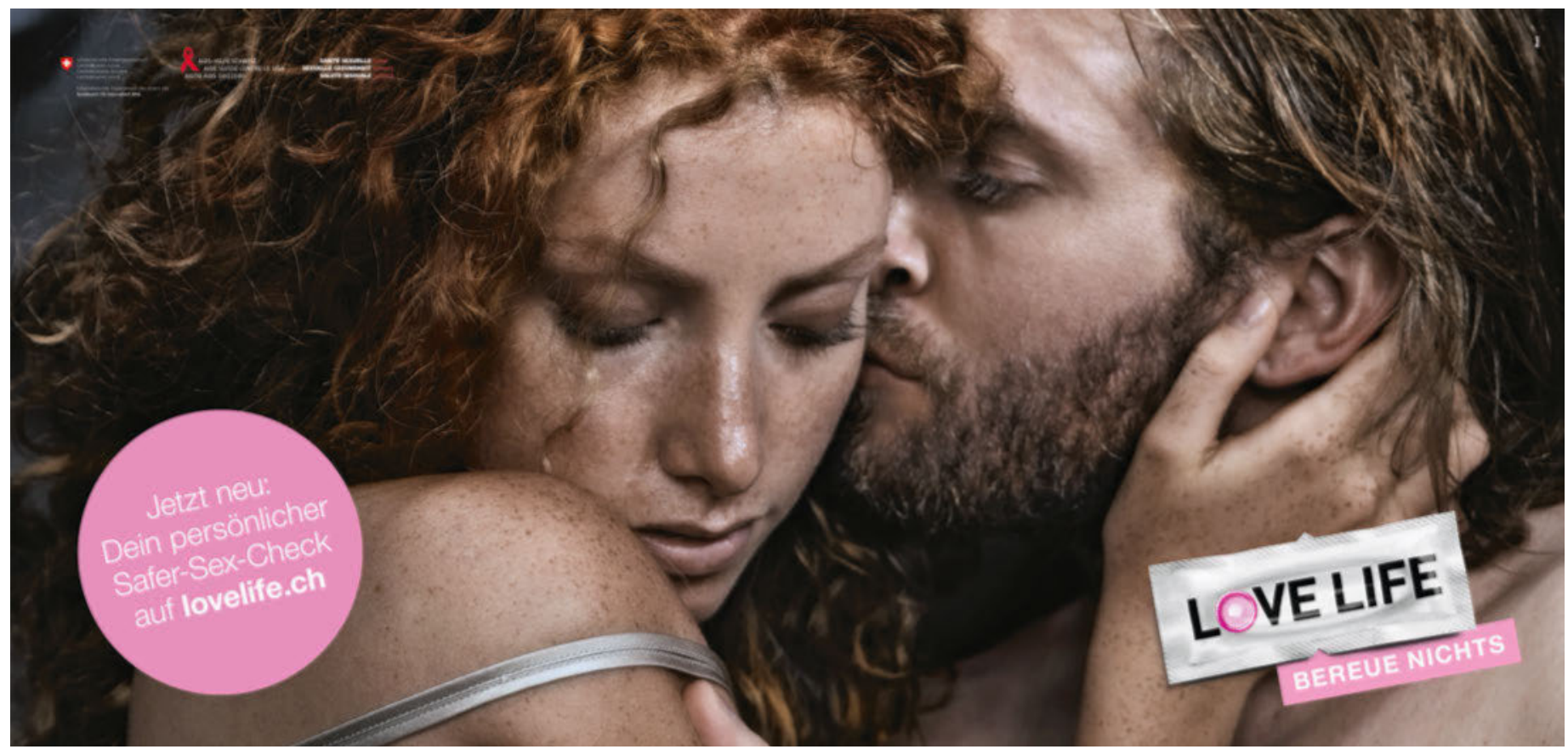

\title{
Aktualisierte Safer-Sex-Regeln und ein individueller Safer-Sex-Check
}

Daniel Koch ${ }^{a}$, Karoline Aebi-Popp ${ }^{b}$, Alexandra Calmyc, Jan Fehr ${ }^{\text {d }}$

${ }^{a}$ Dr. med. MPH; ${ }^{b}$ Dr. med., MSc; ${ }^{\circ}$ Dr. med., PD; ${ }^{d}$ Prof. Dr. med.

Wenn es um Sexualität geht, sind die Safer-Sex-Regeln Thema vieler Beratungsgespräche. Nach 30 Jahren HIV-Prävention war es für das Bundesamt für Gesundheit (BAG) Zeit, die Hauptbotschaften zu überprüfen und zu aktualisieren. Ergebnis ist: Das Kondom bleibt die wichtigste Regel. Die anderen Regeln sind präzisiert und neu in einen Safer-Sex-Check integriert. Der Check ermittelt mit ein paar wenigen Fragen persönlich abgestimmte Empfehlungen. Er weist beispielsweise darauf hin, dass man sich beim Oralsex mit einer sexuell übertragbaren Infektion anstecken kann - auch ohne Sperma oder Blut in den Mund zu nehmen. Nicht neu, aber weiterhin wichtig, sind bei der Diagnose einer sexuell übertragbaren Infektion (STI) die Partnerinformation und die Partnerbehandlung.

Seit der Integration im Jahr 2011 von anderen STI (Syphilis, Gonorrhoe, Chlamydien, virale Hepatitis) ins Nationale HIV-Programm ist Safer Sex nicht mehr nur HIV-Prävention, sondern auch Prävention von anderen sexuell übertragbaren Infektionen. Deshalb sind die Botschaften, was Sex sicherer macht, in den letzten Jah- ren komplexer geworden. Und im Bereich HIV haben neue Erkenntnisse gezeigt, dass es unter gewissen Voraussetzungen zusätzliche Möglichkeiten gibt, sich vor einer HIV-Infektion zu schützen (HIV-Prä-Expositionsprophylaxe) oder eine solche nach einer Risikosituation zu verhindern (HIV-Post-Expositionsprophylaxe). 
Knapp 10 Jahre nach dem «Swiss-Statement» [1] kann bestätigt werden, dass wer dank erfolgreicher HIV-Therapie keine nachweisbare Viruslast mehr aufweist, sexuell niemanden mit dem HI-Virus ansteckt.

Gemeinsam mit Vertreterinnern und Vertretern der Aids-Hilfe Schweiz (AHS), Sexuelle Gesundheit Schweiz (SGCH) und der Eidgenössischen Kommission für sexuelle Gesundheit (EKSG) ist das Bundesamt für Gesundheit (BAG) zum Schluss gekommen, dass es nebst der zentralen Hauptbotschaft - dem Kondomgebrauch - zielführend ist, einen personalisierten Ansatz zu wählen.

\section{Safer-Sex-Regeln: was war - was ist neu}

Bisher galten drei Safer-Sex-Regeln:

1. Eindringender Geschlechtsverkehr - immer mit Kondom (oder Femidom).

2. Kein Sperma und kein Menstruationsblut in den Mund nehmen.

3. Bei Grippesymptomen nach ungeschütztem Sex sowie bei Juckreiz, Brennen, Ausfluss oder anderen Beschwerden im Genitalbereich sofort zum Arzt, zur Ärztin.

Neu werden nur noch zwei Safer-Sex-Regeln kommuniziert:

1. Vaginalsex und Analsex mit Kondom

2. Und weil's jede(r) anders liebt: Mach jetzt deinen persönlichen Safer-Sex-Check auf lovelife.ch

\section{Der individuelle online Safer-Sex-Check}

Das BAG hat gemeinsam mit den erwähnten Institutionen und unter Einbezug weiterer Fachleute und Vertreterinnen und Vertretern von Zielgruppen einen auf www.lovelife.ch online verfügbaren, anonymen SaferSex-Check entwickelt. Nach der Beantwortung weniger Fragen zum persönlichen Sexleben generiert der Check individuelle Safer-Sex-Empfehlungen. Es werden sämtliche Empfehlungen, die für den Schutz vor HIV und STI relevant sind, aufgeführt: Schutz durch Kondom, Schutz durch Impfungen, Informationen zu risikoreichen Sexpraktiken, Beratungsgespräche mit einer Fachperson über HIV und andere STI und die erforderlichen medizinischen Tests. Es wird auch über die Nichtübertragbarkeit von HIV bei einer wirksamen antiretroviralen Therapie als Element der Risikobewertung informiert. Die persönlichen Empfehlungen können zur Gedächtnisstütze oder als Grundlage für ein Beratungsgespräch gespeichert oder ausgedruckt werden. Die Empfehlungen stellen eine Momentaufnahme dar. Wenn sich die persönliche Situation verändert, sollte der Check erneut gemacht werden.

\section{Kondome bleiben wichtig}

Kondome sind ein zuverlässiger Schutz vor HIV. Sie verringern auch das Risiko, sich mit einer anderen STI anzustecken.

\section{Bei Oralsex Übertragung anderer STI als HIV}

Die bisherige Botschaft «Sperma und Blut nicht in den Mund» vermittelt betreffend anderer STI eine falsche Sicherheit. Denn diese können auch ohne Sperma oder Blut im Mund beim Oralsex übertragen werden. $\mathrm{Zu}$ dem ist die Übertragungsgefahr für HIV bei Sperma und Blut im Mund geringer als früher angenommen [2]. Neu vermittelt der Check, dass bei Oralsex die Ansteckung von sexuell übertragbaren Infektionen möglich ist, es sich dabei aber mehrheitlich um heilbare STI handelt und es daher wichtig ist, diese früh zu erkennen und durch eine Therapie auch eine Ansteckung weiterer Personen zu verhindern.

\section{Nicht nur bei Symptomen, sondern auch bei wechselnden Sexualpartnern muss an STI gedacht werden.}

Anstelle des Hinweises in der bisherigen dritten Regel bei Symptomen im Genitalbereich zum Arzt zu gehen, werden neu Personen mit wechselnden Sexualpartnern darauf hingewiesen, einen Arzt, eine Ärztin zu konsultieren. Das Vorgehen, nur symptomatische Personen auf STI zu testen, scheint nicht ausreichend, da es zahlreiche asymptomatische Verläufe gibt. Frauen, die Symptome im Intimbereich haben, haben oft keine STI. Und Frauen ohne Symptome können trotzdem eine STI haben. Auch Männer, die aufnehmenden Analsex praktizieren, haben häufig trotz Infektion keine Symptome [3]. Wechselnde Sexualpartner und nicht nur Symptome sind also ausschlaggebend, sich regelmässig hinsichtlich STI beraten und testen zu lassen.

\section{HIV-Primoinfektion erkennen ist entscheidend}

Fieber und andere grippeähnliche Symptome nach einer Risikosituation können einen wichtigen Hinweis auf eine HIV-Infektion im Anfangsstadium sein (sogenannte Primoinfektion) [4]. Es ist äusserst wichtig, dass 
Korrespondenz:

Daniel Koch, Dr. med. MPH

Leiter Abteilung

Eidgenössisches Departe-

ment des Innern EDI

Bundesamt für Gesundheit

BAG

Abteilung Übertragbare

Krankheiten

Schwarzenburgstrasse 157

CH-3003 Bern

Karoline Aebi-Popp, Dr. med., MSc

Fachärztin Gynäkologie und Geburtshilfe

Universitätsklinik für Infektiologie, Inselspital

CH-3010 Bern

Gruppenpraxis Schönburg

CH-3013 Bern

Dr. med., Alexandra Calmy, PD

Médecin adjointe agrégée

Unité VIH/SIDA

Service de maladies

infectieuses

4 rue Gabrielle Perret Gentil

CH-1211 Genève 14

Prof. Dr. med. Jan Fehr

Departementsleiter Public

Health

Institut für Epidemiologie

Biostatistik und Prävention

Universität Zürich

Hirschengraben 84

CH-8001 Zürich und

Klinik für Infektionskrank

heiten und Spitalhygiene

Universitätsspital Zürich

CH-8091 Zürich eine HIV-Infektion früh erkannt wird: Während der sogenannten HIV-Primoinfektion, also Tage und Wochen nach der Ansteckung, steigt die Viruslast im Blut rasch an. Während dieser Zeit ist die betroffene Person sehr infektiös.

\section{Keine HIV-Transmission unter wirksamer Therapie}

Ein rascher Therapiebeginn ist nicht nur aus individualmedizinischer Sicht wichtig, sondern auch aus Sicht der öffentlichen Gesundheit. Bei einer Person unter erfolgreicher HIV-Therapie steckt sich niemand mehr sexuell an.

\section{Die Prä-Expositionsprophylaxe (PrEP) und die Post-Expositionsprophylaxe (PEP)}

Unter gewissen Voraussetzungen gibt es zusätzlich die Möglichkeit, sich vor einer HIV-Infektion durch eine HIV-Prä-Expositionsprophylaxe zu schützen. Die EKSG hat im Januar 2016 Richtlinien [5] hierzu publiziert. Ebenfalls kann nach einer Risikosituation mit einer HIV-Post-Expositionsprophylaxe [6] eine Ansteckung verhindert werden

\section{STI-Diagnose und Partnerinformation gehören zusammen}

Wird eine STI festgestellt, ist es wichtig, mit dem Patienten, der Patientin die Information an die Sexualpartner zu thematisieren. Den Betroffenen stehen auf www.lovelife.ch/de/hiv-co/partnerinfo/Informationen und unterstützende Hilfsmittel zur Verfügung: Sie können ihre Partner einfach und mithilfe von Textvorlagen oder E-Mails benachrichtigen.

\section{Informationsmaterial zum Safer-Sex- Check}

Für die Bekanntmachung des Safer-Sex-Checks bei Patientinnen/Patienten und bei Klientinnen/Klienten, beispielsweise in Arztpraxen, Fachstellen, Beratungsstellen, Apotheken oder Drogerien, stellt die LOVE LIFEKampagne Promotionsmaterial zur Verfügung: ein Kleinplakat und einen Präsentationsdispenser mit Flyern im Kreditkartenformat. Sie können kostenlos bestellt werden unter: www.bundespublikationen.admin.ch $\rightarrow$ Kampagnen $\rightarrow$ HIV- / STI-Prävention - LOVE LIFE.

Literatur

1 Pietro Vernazza, Bernard Hirschel, Enos Bernasconi, Markus Flepp EKAF: HIV-infizierte Menschen ohne andere STD sind unter wirksamer antiretroviraler Therapie sexuell nicht infektiös https:// saez.ch/de/resource/jf/journal/file/view/article/ saez.2008.13252/2008-05-089.pdf/

2 - Page-Shafer et al. 2002: Risk of HIV infection attributatble to ora sex among men who have sex with men and in the population of men who have sex with men. AIDS 16(17).

- Patel et al. 2014: Per-act HIV transmission risk estimates. AIDS 28(10):1509-1519

- Vittinghoff et al. 1999: Per-Contact Risk of Human Immunodeficiency Transmission between Male Sexual Partners. Am J Epidemio 150(3):306-311

- Baggaley et al. 2008: Systematic review of orogenital HIV-1 transmission probabilities. Int J Epidemiol 37:1255-1265

- Del Romero et al. 2002: Evaluating the risk of HIV transmission through unprotected orogenital sex. AIDS 16 (9):1276-1297

3 CID 41:67-74

4 - Der HIV-Test auf Initiative des Arztes/der Ärztin: https://www. bag.admin.ch/dam/bag/de/dokumente/mt/p-und-p/richtlinienempfehlungen/pict-hiv-test-auf-initiative-des-arztes.pdf.download.pdf/bu-21-15-pict-hiv.pdf und

- Die HIV-Primoinfektion - Hinweise zu Diagnose und Bedeutung https://www.bag.admin.ch/dam/bag/de/dokumente/mt/p-und-p/ richtlinien-empfehlungen/faktenblatt-zu-primoinfektion-2015. pdf.download.pdf/fb-primoinfektion-hiv.pdf

5 Empfehlungen der Eidgenössischen Kommission für sexuelle Gesundheit (EKSG) zur HIV Prä-Expositionsprophylaxe (PrEP) in der Schweiz: https://www.bag.admin.ch/dam/bag/de/dokumente/ $\mathrm{mt} / \mathrm{p}$-und-p/richtlinien-empfehlungen/prep-empfehlungen-dereksg-januar-2016.pdf.download.pdf/bag-04-16-praevention-expo. pdf

6 Notfall HIV-Exposition - PEP kann die richtige Antwort sein: https://www.bag.admin.ch/dam/bag/de/dokumente/mt/p-und-p/ richtlinien-empfehlungen/notfall-hiv-exposition-pep.pdf.download.pdf/bu-48-14-pep-hiv-exposition.pdf

Bildnachweis:

Kampagne LOVE LIFE 